\title{
An Iterative Algorithm for Microwave Tomography Using Modified Gauss-Newton Method
}

\author{
A.K. Kundu ${ }^{1}$, B. Bandyopadhyay ${ }^{1}$ and S. Sanyal ${ }^{2}$ \\ ${ }^{1}$ University of Calcutta /Institute of Radiophysics \& Electronics, Kolkata, India \\ ${ }^{2}$ Tata Institute of Fundamental Research, Mumbai, India
}

\begin{abstract}
An inverse iterative algorithm for microwave imaging based on moment method solution is presented here. A modified Gauss-Newton method has been depicted here to address the nonlinear ill-posed problem. The stabilization term consists of a combination of three weighted discrete derivative operators instead of an Identity matrix as in the Levenberg-Marquardt method based algorithm developed by us. The present algorithm shows a marked improvement over the previous one in the quality of the reconstructed images from synthetic data under noisy condition.
\end{abstract}

Keywords - Regularization matrix, Microwave Tomography, Gauss-Newton Method, Discrete derivative operator

\section{INTRODUCTION}

Microwave tomography techniques for biomedical applications have gained much interest during the last two decades. Immense research is being carried out to qualitatively reconstruct the complex permittivity distribution of the biological media.

Though diffraction tomography has fast numerical algorithm, yet it is marred in strongly inhomogeneous media where Born and Rytov approximations are not valid [1]-[3]. The other methods [4]-[8] based on moment method solutions, are being explored rigorously, but the stability depends on the measurement accuracy due to ill-conditioning of the matrix.

Apart from our first generation algorithms [9] - [10], we had proposed an algorithm [11], which reconstructed the image without any misfit under noise-free environment. However, in noisy condition, the quality of the reconstructed image was rather poor which has been improved by our next algorithm [12]. In this paper, another iterative algorithm based on modified Gauss-Newton method has been proposed which gives a much better result than the previous algorithms under noisy condition.

\section{FoRWARD PROBLEM}

The structure of the forward problem is same as that of our previous works. Here, we have considered a cylindrical object of arbitrary cross section characterized by a complex permittivity distribution $\varepsilon(x, y)$. The object is illuminated by an electromagnetic wave radiated from an open-ended waveguide for which the incident electric field $E^{\text {inc }}$ is parallel to the axis of the cylinder.

Let $E$ represents the total electric field and $E^{S}$ represents the scattered field which is generated by the equivalent electric current radiating in free space so that

$$
\vec{E}=\vec{E}^{i n c}+\vec{E}^{s}
$$

The total electric field can be calculated with an integral representation

$$
\vec{E}(x, y)=\vec{E}^{\text {inc }}(x, y)+\iint_{s} \overrightarrow{J_{s}}(x, y) G\left(x, y ; x^{\prime}, y^{\prime}\right)
$$

$d x^{\prime} d y^{\prime}$

where the Green's function can be given by

$$
G\left(x, y ; x^{\prime}, y^{\prime}\right)=-\frac{j}{4} H_{0}^{2}\left(k \sqrt{\left(x-x^{\prime}\right)^{2}+\left(y-y^{\prime}\right)^{2}}\right)
$$

Here $(x, y)$ and $\left(x^{\prime}, y^{\prime}\right)$ are the observation and source points respectively.

The solution of the forward are carried out by moment method using pulse-basis function and point matching technique [13]. The synthetic data at the receivers is then contaminated with white Gaussian noise with SNR $30 \mathrm{~dB}$ as our main objective is to reconstruct the numerical model under noisy conditions.

\section{INVERSE PROBLEM}

The aim of the inverse problem is to find a stable solution for permittivity distribution $\varepsilon^{*}$ which minimizes the squared error output at the receivers:

$$
\Phi(\varepsilon)=\|E(\varepsilon)-e\|^{2}
$$


where $e \in C^{n}$, the $\mathrm{n}$ electric fields we measure at receiver points, $E: C^{m} \longrightarrow C^{n}$, a function mapping the complex permittivity distribution with $\mathrm{m}$ degrees of freedom into a set of $\mathrm{n}$ approximate electric field observations, and also $\varepsilon \in C^{m}$, the complex permittivity distributions with $\mathrm{m}$ degrees of freedom.

For the minimization of (4), first we compute the GaussNewton direction

$$
\begin{aligned}
& h_{k}=-\left(E^{\prime}\left(\varepsilon_{k}\right)^{\dagger} E^{\prime}\left(\varepsilon_{k}\right)\right)^{-1}\left(E^{\prime}\left(\varepsilon_{k}\right)^{\dagger}\right. \\
& \left.\left(E^{\prime}\left(\varepsilon_{k}\right)-e\right)\right]
\end{aligned}
$$

where $E^{\prime}\left(\varepsilon_{k}\right)$ is the Jacobian matrix evaluated at $\varepsilon_{k}$, $\dagger$ denotes the conjugate transpose.

Due to the inherent instability of ill-poised problems, an iterative method has to be stopped properly to ensure the stability of the iterates. The Gauss-Newton method uses the stabilization term

$$
\left.\left(E\left(\varepsilon_{k}\right)^{\dagger} E^{\prime}\left(\varepsilon_{k}\right)+\lambda_{k} L^{T} L\right)^{-1} \lambda_{k} L^{T} L\left(\varepsilon_{k}-\varepsilon_{a}\right)\right]
$$

leading to the solution

$$
\begin{gathered}
h_{k}=-\left(E^{\prime}\left(\varepsilon_{k}\right)^{\dagger} E^{\prime}\left(\varepsilon_{k}\right)+\lambda_{k} L^{T} L\right)^{-1}\left[E^{\prime}\left(\varepsilon_{k}\right)^{\dagger}\right. \\
\left.\left(E^{\prime}\left(\varepsilon_{k}\right)-e\right)+\lambda_{k} L^{T} L\left(\varepsilon_{k}-\varepsilon_{a}\right)\right]
\end{gathered}
$$

where $\lambda_{k}$ is a monotonically decreasing regularization parameter and $\varepsilon_{a}$ is the assumed permittivity distribution at the start of the iteration. $\mathrm{L}$ is some regularization matrix and is chosen as a discrete approximation of a derivative operator. It is determined by combining several derivative orders (Sobolev norm) and by Cholesky factorization [14] having the form

$$
L^{T} L=\sum_{k=0}^{2} w_{k} L_{k}^{T} L_{k}
$$

where $w_{k} \geq 0$ are some weighting factors such that

$$
\sum_{k=0}^{2} w_{k}=1
$$

The weighting factors are chosen accordance to the peculiarities of the solution.

Instead of conventional iterative procedure by making

$$
\varepsilon_{k+1}=\varepsilon_{k}+a_{k} h_{k}
$$

where $a_{k}$ is the positive step length, we proposed a modified iterative approach. The algorithm leads to an iterative procedure where permittivity distribution at iteration $\mathrm{k}+1$ can be given by

$$
\Delta \varepsilon_{k+1}=\Delta \varepsilon_{k}+a_{k} h_{k}
$$

and

$$
\varepsilon_{k+1}=\varepsilon_{k}+\Delta \varepsilon_{k+1}
$$

where $\varepsilon_{k+1}$ is the permittivity distribution at the $\mathrm{k}+1^{\text {th }}$ iteration.

The iteration is stopped according to the discrepancy principle.

\section{NuMERICAL MODEL}

The theoretical model used to test our algorithm is shown in figure 1

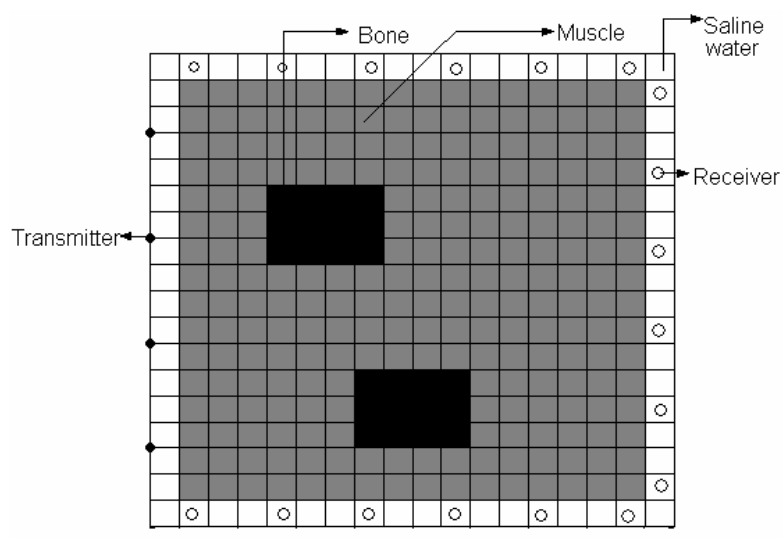

Fig. 1 Theoretical Model

It is a high contrast square biological object $9.6 \mathrm{~cm} \times$ $9.6 \mathrm{~cm}$ consisting of muscle and bone having complex dielectric constants $50-\mathrm{j} 23$ and $8-\mathrm{j} 1.2$ respectively. The object is surrounded by saline water having complex dielectric constant 76-j40.

The object is illuminated with TE fields radiating from an open ended dielectric filled wave guide having sinusoidal aperture field distribution at a frequency of $1 \mathrm{GHz}$. The transmitter is moved along four mutually orthogonal directions. For each of the transmitter positions along a particular transmitting plane, the received fields in the other three orthogonal planes were measured theoretically. The rectangular model together with saline water region is divided into 324 equal square cells $0.6 \mathrm{~cm} \times 0.6 \mathrm{~cm}$. The dimen- 
sions of the bone regions are equal and are given by $1.8 \mathrm{~cm}$ $\times 1.2 \mathrm{~cm}$. During the iterative reconstruction, the complex permittivity values of the cells filled up with saline water were assumed to be known, thus rendering the problem of estimating the complex dielectric constants of the remaining 256 cells.

\section{RESULTS AND DISCUSSION}

To apply the reconstruction algorithm, it was assumed that the biological medium is filled up with muscle only i.e. it was assumed to be homogeneous one having complex dielectric constant $50-\mathrm{j} 23$. The received fields at different receiver locations were computed for each transmitter position.

The algorithm applied here is as follows:

1. The initial values of $\Phi$ and hk are computed from (4) and (5) considering the initial $\lambda_{k}$ to be 0.00001 .

2. Equation (6) and (7) are solved for $\varepsilon_{\text {and }} \Phi_{k+1}$ is evaluated. ak has been chosen as 1 .

3. If $\Phi_{k+1}>\Phi_{k}, \lambda \leftarrow \lambda * 10$ and the control is transferred to 2 .

4. If $\Phi_{k+1}<\Phi_{k}$, then $\lambda \leftarrow \lambda / 10$, the total solution is updated $\varepsilon \leftarrow \varepsilon+\Delta \varepsilon$ and the control is transferred to 2 .

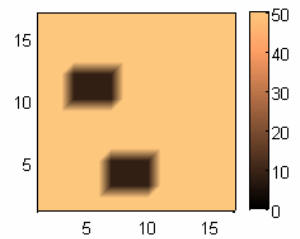

(a)

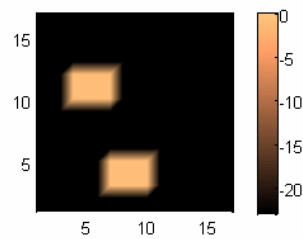

(b)
Fig. 2 Numerical model in terms of (a) real components and (b) imaginary components of the permittivity distribution
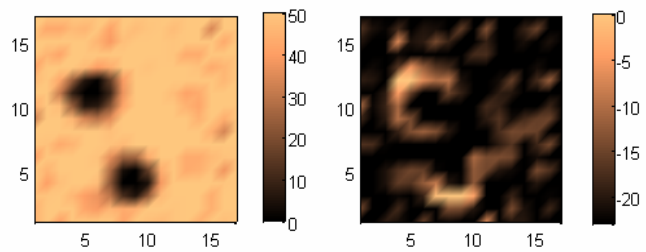

Fig. 3(a) Reconstructed model (under noisy condition) with Levenberg-Merquardt regularization technique
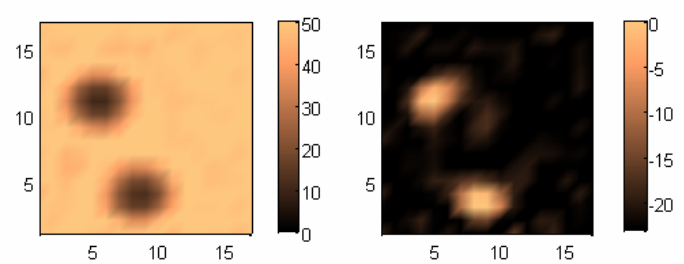

Fig. 3 (b) Reconstructed models (under noisy condition) with Modified Gauss-Newton technique

The only priori information we have used in our algorithm is that the real part of the complex dielectric constant can not be negative and the imaginary part can not be positive. Figure 2 shows the numerical model in terms of the real and imaginary parts. Figure 3 shows the reconstructed model with different algorithms. Figure 3(a) shows the reconstructed model (under noisy condition) with our previous algorithm. Figure 3(b) represents the reconstructed image (under the same noisy condition as the earlier) based on our present algorithm.

The plot of squared error vs. number of iterations is shown in Figure 4.

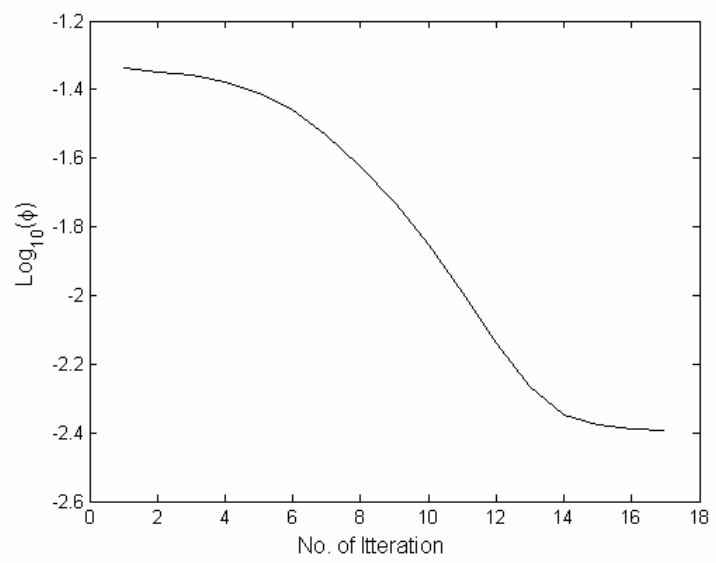

Fig. 4 Plot of squared error vs. number of iterations

We define the Mean Estimation Error as Mean

Mean Estimation Error $=\frac{1}{N} \sum\left|\frac{\varepsilon_{i}-\varepsilon_{i}^{*}}{\varepsilon_{i}^{*}}\right| \times 100$

where $\mathrm{N}$ is the number of cells, $\varepsilon_{i}$ is the estimated permittivity of the $i^{\text {th }}$ cell and $\varepsilon_{i}^{*}$ is the true permittivity of the $\mathrm{i}^{\text {th }}$ cell. The relative errors in the reconstructed image decrease from 39 to $14 \%$ for the real part and 135 to $98 \%$ 
for the imaginary part of the complex dielectric constant as compared with the image shown in Fig. 3.

The result shows that our present iterative algorithm provides us a far better reconstructed image than what we have obtained from our previous algorithm.

\section{REFERENCES}

1. M. Slaney, A.C. Kak and L.E Larsen(1984) Limitations of imaging with first-order diffraction tomography. IEEE Trans. Microwave Theory Tech., vol. .32. No., pp 860-873

2. J.M. Rius, M. Ferando, A Broquetas(1987). Microwave Tomography:. An. Algorithm for Cylindrical Geometries Electron Lett., vol. 23 , pp. $564-565$

3. J. C. Bolomey, C. Pichot, G. Garboriaud (1991). Planar microwave imaging camera for biomedical applications: Critical and prospective analysis of reconstruction algorithms Radio. Sc., vol. 26, n0.. 2, pp 541-549

4. M.N. Ney, A.M. Smith, and S.S. Stuchly(1984) A solution of electromagnetic imaging using pseudoinverse transformation. IEEE Trans. Med. Imag., vol. 3, 155-162, Dec.

5. Y.M. Wang, and W.C. Chew(1989) An iterative solution of the twodimensional electromagnetic inverse scattering problem. Int. J. Imaging Systems and Technology, vol. 1, pp. 100-108,

6. S. Caorsi, G. L. Gragnani, and M. Pastorino(1990) Two-dimensional microwave imaging by a numerical inverse scattering solution. IEEE Transactions on Microwave Theory and Techniques, vol. MTT-38, No. 8, pp. 981-989
7. Fanchosis A, Pichot C(1997) Microwave Imaging- Complex Permittivity reconstruction with a Levenberg- Marquardt Method. IEEE Transaction Antennas and Propagation,vol. 45, No. 2

8. Joachimowicz N, Pichot C, Hugonin J P (1991) Inverse Scattering : An Iterative Numerical Method for Electromagnetic Imaging. IEEE Transaction Antennas and Propagation,vol AP-39, No. 12, pp $1742-1752$

9. A.N. Datta, and B. Bandyopadhyay(1985)An SIRT style reconstruction algorithm for microwave tomography. IEEE Trans. Biomed. Engg., vol. 32, pp 719-723

10. A.N. Datta, and B. Bandyopadhyay(1986) Non-linear extension of moment method solution for microwave tomography. Proc. IEEE. vol. 74 , pp.604 - 605

11. Bandyopadhyay B, Kundu A. K \& Datta A. N(2005)An iterative Algorithm for Microwave Tomography Using Levenberg- Marquardt Regularization Technique. Ind J Pure \& Appl Physics, vol. 43, pp $649-653$

12. Kundu A. K, Bandyopadhyay B(2007) An Iterative Algorithm for Microwave Tomography Using Multiple illuminations of the target. .IEEE Applied Electromagnetics Conference, December 19-20, Kolkata, India

13. J.H. Richmond(1965) Scattering by a dielectric cylinder of arbitrary cross- section shape. IEEE Trans. Antennas and Propagation, vol. 13, No. 3, pp 334-341

14. Eriksson P, J (2000) Analysis and comparison of two linear regularization methods for passive atmospheric observation. Geophysics. vol. 105 , pp 18157-18167 